\title{
ON TWO DIMENSIONAL MARKOV PROCESSES WITH BRANCHING PROPERTY $\left({ }^{(}\right)$
}

\author{
BY \\ SHINZO WATANABE
}

Introduction. A continuous state branching Markov process (C.B.P.) was introduced by Jirina [8] and recently Lamperti [10] determined all such processes on the half line. (A quite similar result was obtained independently by the author.) This class of Markov processes contains as a special case the diffusion processes (which we shall call Feller's diffusions) studied by Feller [2]. The main objective of the present paper is to extend Lamperti's result to multi-dimensional case. For simplicity we shall consider the case of 2-dimensions though many arguments can be carried over to the case of higher dimensions $\left({ }^{2}\right)$. In Theorem 2 below we shall characterize all C.B.P.'s in the first quadrant of a plane and construct them. Our construction is in an analytic way, by a similar construction given in Ikeda, Nagasawa and Watanabe [5], through backward equations (or in the terminology of [5] through $S$-equations) for a simpler case and then in the general case by a limiting procedure.

A special attention will be paid to the case of diffusions. We shall show that these diffusions can be obtained as a unique solution of a stochastic equation of Ito (Theorem 3). This fact may be of some interest since the solutions of a stochastic equation with coefficients Hölder continuous of exponent $1 / 2$ (which is our case) are not known to be unique in general. Next we shall examine the behavior of sample functions near the boundaries ( $x_{1}$-axis or $x_{2}$-axis). We shall explain, for instance, the case of $x_{1}$-axis. There are two completely different types of behaviors. In the first case $x_{1}$-axis acts as a pure exit boundary: when a sample function reaches the $x_{1}$-axis then it remains on it moving as a one-dimensional Feller diffusion up to the time when it hits the origin and then it is stopped. In the second case, there is a point $x_{0}$ on $x_{1}$-axis such that $\Sigma_{2}=\left(0, x_{0}\right)$ acts as a reflecting boundary and $\Sigma_{1}=$ $\left(x_{0}, \infty\right)$ acts as a pure entrance boundary (Theorem 4 and Corollaries).

1. Definitions and the main theorem. Let $D^{\circ}=\left\{x=\left(x_{1}, x_{2}\right): x_{1}>0, x_{2}>0\right\}$, $D=\left\{x=\left(x_{1}, x_{2}\right): x_{1} \geqq 0, x_{2} \geqq 0\right\}$ and $\bar{D}=D \cup\{\Delta\}$ be the one-point compactification of $D$.

Received by the editors September 25, 1967.

(1) Research supported in part under contract NOO14-67-A-0112-0115 at Stanford University, Stanford, California.

$\left({ }^{2}\right)$ Also a similar result can be obtained for more general domains, e.g., upper half plane or whole plane (in the latter case every process is a deterministic diffusion). 
Definition 1.1 $\left({ }^{3}\right)$. A Markov process $X=\left(x_{t}, P_{x}\right)$ on $\bar{D}$ is called a continuous state branching process (C.B.P.) if it has $\Delta$ as a trap and satisfies

$$
E_{x}\left(e^{-\lambda \cdot x_{t}}\right)=E_{(1,0)}\left(e^{-\lambda \cdot x_{t}}\right)^{x_{1}} E_{(0,1)}\left(e^{-\lambda \cdot x_{t}}\right)^{x_{2}}
$$

for every $\lambda=\left(\lambda_{1}, \lambda_{2}\right) \gg 0$ and $x=\left(x_{1}, x_{2}\right) \in D$. The property $(1.1)$ is called the branching property.

Definition 1.2. A C.B.P. $X=\left(x_{t}, P_{x}\right)$ is called regular if both $E_{(1,0)}\left(e^{-\lambda \cdot x_{t}}\right)$ and $E_{(0,1)}\left(e^{-\lambda \cdot x_{t}}\right)$ are differentiable in $t$ at $t=0$ for each fixed $\lambda \gg 0$.

Let $\Psi$ be the set of all functions $\psi(\lambda), \lambda \geqq 0$, of the form

$$
\psi(\lambda)=c_{0}+c_{1} \lambda_{1}+c_{2} \lambda_{2}+\int_{D}\left(1-e^{-\lambda \cdot u}\right) n(d u)
$$

where $c_{i} \geqq 0, i=0,1,2$ and $n(d u)$ is a nonnegative measure on $D-\{O\}\left(^{4}\right)$ such that

$$
\int_{D} \frac{|u|}{1+|u|} n(d u)<\infty
$$

i.e., $\psi(\lambda) \in \Psi$ if and only if $e^{-\psi(\lambda)}$ is the Laplace transform of a substochastic infinitely divisible measure $P$ on $D$. Let

$$
\Psi^{2}=\Psi \times \Psi=\left\{\psi(\lambda)=\left(\psi_{1}(\lambda), \psi_{2}(\lambda)\right) ; \psi_{i}(\lambda) \in \Psi, i=1,2\right\}
$$

then there is a one-to-one correspondence between $\psi \in \Psi^{2}$ and a pair $\left(P_{1}, P_{2}\right)$ of substochastic infinitely divisible measures on $D$. It is easy to see that if $\psi_{1}$ and $\psi_{2}$ are in $\Psi^{2}$ then $\psi_{1}\left(\psi_{2}\right) \in \Psi^{2}$ where $\psi_{1}\left(\psi_{2}\right)$ is defined by

$$
\psi_{1}\left(\psi_{2}\right)(\lambda)=\left(\psi_{1}^{(1)}\left(\psi_{2}(\lambda)\right), \psi_{2}^{(1)}\left(\psi_{2}(\lambda)\right)\right)
$$

if $\psi_{1}=\left(\psi_{1}^{(1)}, \psi_{2}^{(1)}\right)$. In fact for every $x=\left(x_{1}, x_{2}\right) \in D$ there exists a unique substochastic infinitely divisible measure $P_{x}(d y)$ on $D$ such that

$$
\exp \left\{-x_{1} \psi_{1}^{(2)}(\lambda)-x_{2} \psi_{2}^{(2)}(\lambda)\right\}=\int_{D} e^{-\lambda \cdot y} P_{x}(d y)\left({ }^{5}\right)
$$

Let $Q_{i}(d y)(i=1,2)$ be substochastic infinitely divisible measures on $D$ defined by

$$
\exp \left(-\psi_{i}^{(1)}(\lambda)\right)=\int_{D} e^{-\lambda \cdot y} Q_{i}(d y)
$$

Then $\psi_{1}\left(\psi_{2}\right)$ corresponds to the pair $\left(P_{1}, P_{2}\right)$ of infinitely divisible measures defined by

$$
P_{i}(d y)=\int_{D} Q_{i}(d x) P_{x}(d y), \quad i=1,2 .
$$

$\left(^{3}\right) \lambda \gg 0\left(\lambda=\left(\lambda_{1}, \lambda_{2}\right)\right)$ means $\lambda_{l}>0^{\prime}(i=1,2) . \lambda \geqq 0$ means $\lambda_{l} \geqq 0(i=1,2) . \lambda \cdot x=\lambda_{1} x_{1}+\lambda_{2} x_{2}$ for $\lambda=\left(\lambda_{1}, \lambda_{2}\right)$ and $x=\left(x_{1}, x_{2}\right)$.

( $\left.{ }^{4}\right) O$ denotes the origin.

(5) $\psi_{2}=\left(\psi_{1}^{(2)}, \psi_{2}^{(2)}\right)$. 
Definition 1.3. A one parameter family $\left\{\boldsymbol{\psi}_{t}\right\}_{t \in[0, \infty)}$ of elements in $\Psi^{2}$ is called a $\Psi^{2}$-semigroup if

$$
\psi_{t+s}=\psi_{t}\left(\psi_{s}\right), \quad \psi_{0}(\lambda)=\lambda .
$$

Definition 1.4. A $\Psi^{2}$-semigroup $\left\{\Psi_{t}\right\}$ is called regular if for every $\lambda \gg 0, \Psi_{t}(\lambda)$ is differentiable in $t$ at $t=0$.

Let $X=\left(x_{t}, P_{x}\right)$ be a C.B.P. and let $P(t, x, d y)$ be the transition probability of $X$. If we set $P_{1}(t, d y)=P(t,(1,0), d y)$ and $P_{2}(t, d y)=P(t,(0,1), d y)$ then

$$
\int_{D} e^{-\lambda \cdot y} P(t, x, d y)=\exp \left\{-\psi_{1}(t, \lambda) x_{1}-\psi_{2}(t, \lambda) x_{2}\right\}
$$

where

$$
\exp \left(-\psi_{i}(t, \lambda)\right)=\int_{D} e^{-\lambda \cdot y} P_{i}(t, d y), \quad i=1,2 .
$$

Then $\psi_{t}(\lambda)=\left(\psi_{1}(t, \lambda), \psi_{2}(t, \lambda)\right) \in \Psi^{2}$ for every $t \geqq 0$ and by the semigroup property of $P(t, x, d y)$ it is easy to see that $\psi_{t+s}=\psi_{t}\left(\psi_{s}\right)$, i.e., $\left\{\Psi_{t}\right\}_{t \in[0, \infty)}$ is a $\Psi^{2}$-semigroup. Conversely given a $\Psi^{2}$-semigroup $\left\{\Psi_{t}\right\}$, if we define $P(t, x, d y)$ by (1.6) then it is a substochastic kernel and by the semigroup property of $\Psi_{t}$ we have

$$
P(t+s, x, d y)=\int_{D} P(t, x, d z) P(s, z, d y) .
$$

Hence $\{P(t, x, d y)\}$ defines a unique Markov process on $\bar{D}=D \cup\{\Delta\}$ with $\bar{D}$ as a trap. Thus we have the following

THEOREM 1. There is a one-to-one correspondence between the C.B.P.' $X=\left(x_{t}, P_{x}\right)$, and the $\Psi^{2}$-semigroups $\left\{\Psi_{t}\right\}$. The correspondence is given by

$$
E_{x}\left(e^{-\lambda \cdot x_{t}}\right)=\exp \left\{-x_{1} \psi_{1}(t, \lambda)-x_{2} \psi_{2}(t, \lambda)\right\} .
$$

Furthermore $X$ is regular if and only if $\left\{\psi_{t}\right\}$ is regular.

Let $\xi_{1}(x)$ and $\xi_{2}(x) \in C_{0}^{\infty}(D)\left({ }^{6}\right)$ such that $\xi_{1}(x)=x_{1}$ and $\xi_{2}(x)=x_{2}$ on some neighborhood $U$ of the origin $O$. The main theorem is the following:

Theorem 2. Let $X=\left(x_{t}, P_{x}\right)$ be a regular C.B.P. Then the semigroup $T_{t}$ of the process $X$ is a strongly continuous nonnegative contraction semigroup on $C_{0}(D)\left({ }^{6}\right)$ such that, if $A$ is the infinitesimal generator in Hille-Yosida sense of $T_{t}$, we have

(i) $C_{0}^{2}(D) \subset D(A)\left({ }^{6}\right)$ and

(ii) for $f \in C_{0}^{2}(D)$

$\left({ }^{6}\right) C_{0}(D)=\left\{f(x)\right.$ : continuous on $D$ such that $\left.\lim _{|x| \rightarrow \infty} f(x)=0\right\}, C_{0}^{n}(D)=\left\{f(x) \in C_{0}(D)\right.$; all the derivatives up to $n$th order are in $\left.C_{0}(D)\right\}$ and $C_{0}^{\infty}(D)=\bigcap_{n} C_{0}^{n}(D)$. 


$$
\begin{aligned}
A f(x)= & \alpha^{2} x_{1} f_{11}(x)+\beta^{2} x_{2} f_{22}(x)+\left(a x_{1}+b x_{2}\right) f_{1}(x) \\
& +\left(c x_{1}+d x_{2}\right) f_{2}(x)-\gamma x_{1} f(x)-\delta x_{2} f(x) \\
& +x_{1} \int_{D}\left\{f(x+y)-f(x)-\xi_{1}(y) f_{1}(x)\right\} n_{1}(d y) \\
& +x_{2} \int_{D}\left\{f(x+y)-f(x)-\xi_{2}(y) f_{2}(x)\right\} n_{2}(d y)\left(7^{7}\right)
\end{aligned}
$$

where $\alpha, \beta, \gamma, \delta, a, b, c, d$ are constants such that

$$
\gamma \geqq 0, \quad \delta \geqq 0, \quad b \geqq 0, \quad c \geqq 0
$$

and $n_{1}, n_{2}$ are nonnegative measures on $D-\{O\}$ such that

$$
\begin{aligned}
\int_{U}\left\{\xi_{1}^{2}(y)+\xi_{2}(y)\right\} n_{1}(d y)+\int_{U}\left\{\xi_{1}(y)+\right. & \left.\xi_{2}^{2}(y)\right\} n_{2}(d y) \\
& +n_{1}(D-U)+n_{2}(D-U)<\infty .
\end{aligned}
$$

Conversely given $a, b, c, d, \alpha, \beta, \gamma, \delta, n_{1}$ and $n_{2}$ with the conditions (1.10) and (1.11) there exists a unique Markov process $X=\left(x_{t}, P_{x}\right)$ with the semigroup $T_{t}$ satisfying (i) and (ii) and further $X$ is a regular C.B.P.

If we set

$$
\begin{gathered}
E_{(1,0)}\left(e^{-\lambda \cdot x_{t}}\right)=e^{-\psi_{1}(t, \lambda)}, \quad E_{(0,1)}\left(e^{-\lambda \cdot x_{t}}\right)=e^{-\psi_{2}(t, \lambda)}, \\
h_{1}(\lambda)=-\alpha^{2} \lambda_{1}+\left(a \lambda_{1}+c \lambda_{2}\right)+\gamma-\int_{D}\left(e^{-\lambda y}-1+\lambda_{1} \xi_{1}(y)\right) n_{1}(d y), \\
h_{2}(\lambda)=-\beta^{2} \lambda_{1}+\left(b \lambda_{1}+d \lambda_{2}\right)+\delta-\int_{D}\left(e^{-\lambda y}-1+\lambda_{2} \xi_{2}(y)\right) n_{2}(d y),
\end{gathered}
$$

and

$$
E_{x}\left(e^{-\lambda \cdot x_{t}}\right)=F(t, \lambda ; x)
$$

then $\psi_{t}=\left(\psi_{1}(t, \lambda), \psi_{2}(t, \lambda)\right)$ satisfies the backward equation

$$
d \psi_{1} / d t=h_{1}\left(\psi_{t}\right), \quad d \psi_{2} / d t=h_{2}\left(\psi_{t}\right), \quad \psi_{0+}=\lambda,
$$

and $F(t, \lambda ; x)$ satisfies the forward equation

$$
\frac{\partial F}{\partial t}=h_{1}(\lambda) \frac{\partial F}{\partial \lambda_{1}}+h_{2}(\lambda) \frac{\partial F}{\partial \lambda_{2}} ; \quad F(0+, \lambda ; x)=e^{-\lambda \cdot x}
$$

By the correspondence established in Theorem 1 this theorem can be stated in the following purely analytical form:

THEOREM $2^{\prime}$. Let $\Psi_{t}=\left(\psi_{1}(t, \lambda), \psi_{2}(t, \lambda)\right)$ be a regular $\Psi^{2}$-semigroup. Then there exist $a, b, c, d, \alpha, \beta, \gamma, \delta, n_{1}$ and $n_{2}$ satisfying the conditions (1.10) and (1.11) such that it is given as a solution of the backward equation (1.15) for $h_{1}$ and $h_{2}$ defined by (1.13).

$\left.{ }^{7}\right) f_{i j}(x)=\partial^{2} f / \partial x_{i} \partial x_{j}$ and $f_{i}(x)=\partial f / \partial x_{i}, i=1,2$ 
Conversely given such $a, b, c, d, \alpha, \beta, \gamma, \delta, n_{1}$ and $n_{2}$, the solution of the backward equation is unique and defines a $\Psi^{2}$-semigroup.

\section{Proof of Theorem 2.}

LeMma 1. Let $\psi_{n} \in \Psi$ and $\psi_{n} \Rightarrow \psi\left(\left(^{8}\right)\right.$ on a domain $U \Subset \Lambda=\left\{\lambda=\left(\lambda_{1}, \lambda_{2}\right): \lambda \geqq 0\right\}\left({ }^{9}\right)$. Then $\psi \in \Psi$. (To be precise there exists a unique extension of $\Psi$ onto $\Lambda$ so that it is an element of $\Psi$.)

Proof. This lemma may be well known but we shall give a standard proof for the completeness. $\psi(\lambda) \in \Psi$ has the form

$$
\begin{aligned}
\psi(\lambda) & =c_{0}+c_{1} \lambda_{1}+c_{2} \lambda_{2}+\int_{D}\left(1-e^{-\lambda \cdot u}\right) n(d u) \\
& =\int_{\Omega} \xi(\lambda ; r, \theta) \tilde{n}(d r d \theta)
\end{aligned}
$$

for some bounded measure $\tilde{n}(d r d \theta)$ on $\Omega$ where

$$
\begin{gathered}
\Omega=[0, \infty] \times[0, \pi / 2] \\
\xi(\lambda ; r, \theta)=\left\{1-\exp \left(-\lambda_{1} r \cos \theta-\lambda_{2} r \sin \theta\right)\right\} \\
\times(1+r \cos \theta+r \sin \theta)(r \cos \theta+r \sin \theta)^{-1} .
\end{gathered}
$$

Clearly we have

$$
c_{0}=\int_{\{\infty\} \times[0, \pi / 2]} \tilde{n}(d r d \theta), \quad c_{1}=\int_{\{0\} \times[0, \pi / 2]} \frac{\cos \theta}{\cos \theta+\sin \theta} \tilde{n}(d r d \theta)
$$

and

$$
c_{2}=\int_{\{0\} \times[0, \pi / 2]} \frac{\sin \theta}{\cos \theta+\sin \theta} \tilde{n}(d r d \theta) .
$$

Also it is easy to see that for any nonnegative bounded measure $\tilde{n}$ on $\Omega$

$$
\psi(\lambda)=\int_{\Omega} \xi(\lambda ; r, \theta) \tilde{n}(d r d \theta)
$$

is an element in $\Psi$. Note also that for fixed $\lambda_{0} \in U$,

$$
d_{1} \leqq \xi\left(\lambda_{0} ; r, \theta\right) \leqq d_{2} \quad \text { for all }(r, \theta) \in \Omega
$$

for some constants $0<d_{1}<d_{2}$. Now let $\psi_{n}(\lambda) \Rightarrow \psi(\lambda)$ on $U$. Then $\sup _{n} \psi_{n}\left(\lambda_{0}\right)<\infty$ and hence by $(2.1) \sup _{n} \tilde{n}_{n}(\Omega)<\infty$. Hence some subsequence $\left\{\tilde{n}_{n_{l}}\right\}$ of $\left\{\tilde{n}_{n}\right\}$ converges weakly to a bounded measure $\tilde{n}$, then clearly

$$
\psi_{n_{i}}(\lambda) \rightarrow \psi(\lambda)=\int_{\Omega} \xi(\lambda ; r, \theta) \tilde{n}(d r d \theta) \in \Psi
$$

$\left(^{8}\right) \psi_{n} \Rightarrow \psi$ means the uniform convergence.

( $\left.{ }^{9}\right) U \subset \Lambda$ means $U$ is a bounded domain such that $\rho(U ; \partial \Lambda)>0$ where $\rho$ is the Euclidean distance. 
LEMMA 2. Let $h_{n}(\lambda)=\left(h_{1}^{(n)}(\lambda), h_{2}^{(n)}(\lambda)\right), n=1,2, \ldots$, and $h(\lambda)=\left(h_{1}(\lambda), h_{2}(\lambda)\right)$ be functions on $\Lambda=\left\{\lambda=\left(\lambda_{1}, \lambda_{2}\right) ; \lambda_{i} \geqq 0\right\}$ such that for some $V \Subset \Lambda, h_{n} \Rightarrow h$ on $V$ and each $h_{n}$ and $h$ are Lipschitz continuous on $V$. Let $\psi_{t}^{(n)}(\lambda)$ and $\psi_{t}(\lambda)$ be solutions of

$$
d \psi_{t}^{(n)} / d t=h_{n}\left(\Psi_{t}^{(n)}\right), \quad \Psi_{0+}^{(n)}=\lambda
$$

and

$$
d \psi_{t} / d t=h\left(\psi_{t}\right), \quad \psi_{0+}=\lambda
$$

respectively. Then there exists $V_{0} \Subset V$ and $t_{0}>0$ such that $\Psi_{t}^{(n)}(\lambda) \Rightarrow \Psi_{t}(\lambda)$ on $(t, \lambda)$ $\in\left[0, t_{0}\right] \times V_{0}$.

Proof. Let $h(\lambda), \tilde{h}(\lambda)$ be Lipschitz continuous in $V$ such that

$$
\sup _{\lambda \in V}|h(\lambda)-\tilde{h}(\lambda)|<\varepsilon
$$

and

$$
\sup _{\lambda, u \in V}|h(\lambda)-h(\mu)| \cdot|\lambda-\mu|^{-1} \leqq A\left({ }^{10}\right) .
$$

Set

$$
B=\sup _{\lambda \in V}|h(\lambda)|+\varepsilon .
$$

We claim that if $\psi_{t}$ and $\tilde{\psi}_{t}$ are the solutions of

$$
\Psi_{t}(\lambda)=\lambda+\int_{0}^{t} h\left(\Psi_{s}(\lambda)\right) d s
$$

and

$$
\tilde{\psi}_{t}(\lambda)=\lambda+\int_{0}^{t} \tilde{h}\left(\tilde{\Psi}_{s}(\lambda)\right) d s
$$

respectively then

$$
\sup _{0 \leqq t \leqq t_{0}} \sup _{\lambda \in V_{0}}\left|\Psi_{t}(\lambda)-\tilde{\Psi}_{t}(\lambda)\right| \leqq \frac{\varepsilon}{A} \exp \left(A t_{0}\right)
$$

where $V_{0}$ is a domain such that $V_{0} \Subset V$ and $t_{0}>0$ is such that

$$
\rho\left(V_{0} ; \partial V\right)>B t_{0} \text {. }
$$

In fact, set $\psi_{t}^{(0)}=\tilde{\psi}_{t}^{(0)}=\lambda$ and define $\psi_{t}^{(n)}$ and $\tilde{\psi}_{t}^{(n)}$ successively by

$$
\psi_{t}^{(n)}=\lambda+\int_{0}^{t} h\left(\psi_{s}^{(n-1)}\right) d s
$$

and

$$
\tilde{\psi}_{t}^{(n)}=\lambda+\int_{0}^{t} \tilde{h}\left(\tilde{\Psi}^{(n-1)}\right) d s .
$$

(10) $A$ is a positive constant. 
It is clear that if $(t, \lambda) \in\left[0, t_{0}\right] \times V_{0}$ then $\psi_{t}^{(n)}$ and $\tilde{\psi}_{t}^{(n)} \in V$ and $\psi_{t}^{(n)} \Rightarrow \psi_{t}$ and $\tilde{\Psi}_{t}^{(n)} \Rightarrow \tilde{\Psi}_{t}$. We shall prove that for $0 \leqq t \leqq t_{0}$

$$
\sup _{0 \leqq s \leqq t} \sup _{\lambda \in V_{0}}\left|\Psi_{s}^{(n)}(\lambda)-\tilde{\Psi}_{s}^{(n)}(\lambda)\right| \leqq \frac{\varepsilon}{A}\left[A t+\frac{(A t)^{2}}{2 !}+\cdots+\frac{(A t)^{n}}{n !}\right] .
$$

In fact, (2.10) is trivial for $n=0$ and if we assume that (2.10) is valid for $n=0,1,2$, $\ldots, n$ then for $\lambda \in V_{0}$ and $0 \leqq t \leqq t_{0}$,

$$
\begin{aligned}
\left|\Psi_{t}^{(n+1)}(\lambda)-\tilde{\Psi}_{t}^{(n+1)}(\lambda)\right| & \leqq \int_{0}^{t}\left|h\left(\Psi_{s}^{(n)}\right)-\tilde{h}\left(\tilde{\Psi}_{s}^{(n)}\right)\right| d s \\
& \leqq \int_{0}^{t}\left|h\left(\Psi_{s}^{(n)}\right)-h\left(\tilde{\Psi}_{s}^{(n)}\right)\right| d s+\int_{0}^{t} \mid\left(h\left(\tilde{\Psi}_{s}^{(n)}\right)-\tilde{h}\left(\tilde{\Psi}_{s}^{(n)}\right) \mid d s\right. \\
& \leqq A \int_{0}^{t}\left|\Psi_{s}^{(n)}(\lambda)-\tilde{\Psi}_{s}^{(n)}(\lambda)\right| d s+\varepsilon t \\
& \leqq \varepsilon \int_{0}^{t}\left[A s+\frac{(A s)^{2}}{2 !}+\cdots+\frac{(A s)^{n}}{n !}\right] d s+\varepsilon t \\
& =\frac{\varepsilon}{A}\left[A t+\frac{(A t)^{2}}{2 !}+\cdots+\frac{(A t)^{n+1}}{(n+1) !}\right]
\end{aligned}
$$

Therefore (2.10) holds for every $n$. Letting $n \rightarrow \infty$ we have (2.9). The assertion of the lemma is clear from the inequality (2.9).

Lemma 3. Let $h(\lambda)=\left(h_{1}(\lambda), h_{2}(\lambda)\right)$ be a function defined on $\Lambda=\{\lambda: \lambda \geqq 0\}$, Lipschitz continuous on a domain $V \Subset \Lambda$. Let $F(t, \lambda)$ be a continuous function of $(t, \lambda) \in[0, \infty)$ $\times V$, (real) analytic in $\lambda \in V$ for each fixed $t$ and satisfy

$$
\frac{\partial F}{\partial t}=h_{1}(\lambda) \frac{\partial F}{\partial \lambda_{1}}+h_{2}(\lambda) \frac{\partial F}{\partial \lambda_{2}}, \quad F(0, \lambda)=0 .
$$

Then $F(t, \lambda)=0$ for all $(t, \lambda) \in[0, \infty) \times V$.

Proof. It is sufficient to prove $F(t, \lambda)=0$ for all $(t, \lambda) \in\left[0, t_{0}\right] \times V$ for some $t_{0}$, since then $\tilde{F}(t, \lambda)=F\left(t+t_{0}, \lambda\right)$ is also a solution of $(2.11)$ and hence $\tilde{F}(t, \lambda)=0$ for all $(t, \lambda) \in\left[0, t_{0}\right] \times V$ i.e., $F(t, \lambda)=0$ for all $(t, \lambda) \in\left[0,2 t_{0}\right] \times V$. Continuing this process we have $F(t, \lambda)=0$ for all $(t, \lambda) \in[0, \infty) \times V$.

Since $F(t, \lambda)$ is analytic in $\lambda \in V$ for each $t$ it is sufficient to show that $F(t, \lambda)=0$ for all $(t, \lambda) \in\left[0, t_{0}\right] \times V_{1}$ for some $V_{1} \Subset V$. Let $\psi_{t}(\lambda)=\left(\psi_{1}(t, \lambda), \psi_{2}(t, \lambda)\right)$ be the solution of

$$
d \psi_{t} / d t=h\left(\psi_{t}\right), \quad \psi_{0}(\lambda)=\lambda .
$$

Then there exists $t_{0}$ and $V_{1} \Subset V$ such that $\psi_{t}(\lambda) \in V$ for all $(t, \lambda) \in\left[0, t_{0}\right] \times V_{1}$. Set $G(\sigma, \lambda)=F\left(t-\sigma, \psi_{\sigma}(\lambda)\right), 0 \leqq \sigma \leqq t \leqq t_{0}, \lambda \in V_{1}$ then

$$
\begin{aligned}
\frac{d G}{d \sigma} & =-\frac{\partial F}{\partial t}\left(t-\sigma, \Psi_{\sigma}(\lambda)\right)+\frac{\partial F}{\partial \lambda_{1}}\left(t-\sigma, \Psi_{\sigma}\right) \frac{d \psi_{1}(\sigma, \lambda)}{d \sigma}+\frac{\partial F}{\partial \lambda_{2}}\left(t-\sigma, \Psi_{\sigma}(\lambda)\right) \frac{d \psi_{2}(\sigma, \lambda)}{d \sigma} \\
& =\left.\left(-\frac{\partial F}{\partial t}+h_{1} \frac{\partial F}{\partial \lambda}+h_{2} \frac{\partial F}{\partial \lambda}\right)\right|_{\lambda=\psi_{\sigma}} ^{t=t-\sigma}=0
\end{aligned}
$$


and hence $G(\sigma, \lambda)$ is constant in $\sigma$. In particular we have $F(t, \lambda)=G(0, \lambda)=G(t, \lambda)$ $=F\left(0, \psi_{t}(\lambda)\right)=0$ and therefore $F(t, \lambda)=0$ for all $(t, \lambda) \in\left[0, t_{0}\right] \times V_{1}$.

Now we shall prove Theorem 2. First, let $X=\left(x_{t}, P_{x}\right)$ be a regular C.B.P. and let $T_{t}$ be its semigroup. It is easy to see that $T_{t}$ is a semigroup on $C_{0}$. Let $A$ be the infinitesimal generator in Hille-Yosida sense of $T_{t}$ and let $D(A)$ be the domain of $A$. Set

$$
f_{\lambda}(x)=e^{-\lambda \cdot x}, \quad \lambda=\left(\lambda_{1}, \lambda_{2}\right) \geqq 0, \quad x=\left(x_{1}, x_{2}\right) \in D .
$$

Then

$$
T_{t} f_{\lambda}(x)=\left(T_{t} f_{\lambda}(1,0)\right)^{x_{1}}\left(T_{t} f_{\lambda}(0,1)\right)^{x_{2}}
$$

By the regularity of $X$ and (2.13), $T_{t} f_{\lambda}(x)$ is differentiable in $t$ at $t=0$ for each $\lambda \gg 0$ and noting that, for some $t_{0}>0$,

$$
\sup _{0 \leqq t \leqq t_{0}} T_{t} f_{\lambda}(1,0)<1 \text { and } \sup _{0 \leqq t \leqq t_{0}} T_{t} f_{\lambda}(0,1)<1
$$

it is easy to see that $\left(T_{t} f_{\lambda}(x)-f_{\lambda}(x)\right) / t$ converges uniformly in $x$ when $t \downarrow 0$. Hence $\left\{f_{\lambda}: \lambda \gg 0\right\} \subset D(A)$. By (2.13) we have also

$$
A f_{\lambda}(x)=\lim _{t \downarrow 0} \frac{T_{t} f_{\lambda}(x)-f_{\lambda}(x)}{t}=e^{-\lambda \cdot x}\left\{e^{\lambda_{1}} A f_{\lambda}(1,0) x_{1}+e^{\lambda_{2}} A f_{\lambda}(0,1) x_{2}\right\} .
$$

We shall now determine the expression of $A f_{\lambda}(1,0)$ and $A f_{\lambda}(0,1)$. More generally we shall determine the expression of $A f_{\lambda}$ at a boundary point $x_{0}$ of $D$ except origin $O\left({ }^{11}\right)$. Let $x_{0}=\left(x_{1}^{0}, 0\right)$ where $x_{1}^{0}>0$ and $V$ be a neighborhood in $D$ of $x_{0}$. Let $\xi_{1}(x), \xi_{2}(x) \in C_{0}^{\infty}\left(R^{n}\right)\left({ }^{12}\right)$ such that $\xi_{1}(x)=x_{1}$ and $\xi_{2}(x)=x_{2}$ on some neighborhood $U$ of the origin. Let $D_{x_{0}}$ (resp. $V_{x_{0}}$ ) $=\left\{x=y-x_{0} ; y \in D\right.$ (resp. $y \in V$ ) $\}$ and we assume $V_{x_{0}} \subset U$. By a result due to Venttsel' [15], there exists $\alpha^{2}\left(x_{0}\right), a\left(x_{0}\right), c\left(x_{0}\right) \geqq 0$, $\gamma\left(x_{0}\right) \geqq 0, \sigma\left(x_{0}\right) \leqq 0$ and a nonnegative measure $n_{x_{0}}(d y)$ on $D_{x_{0}}-\{O\}$ with

$$
\int_{V_{x_{0}}}\left\{\xi_{1}(y)^{2}+\xi_{2}(y)\right\} n_{x_{0}}(d y)+n_{x_{0}}\left(D_{x_{0}}-V_{x_{0}}\right)<\infty
$$

such that for every $f \in D(A) \cap C^{2}(V)$,

$$
\begin{aligned}
L f\left(x_{0}\right) \equiv & \alpha^{2}\left(x_{0}\right) f_{11}\left(x_{0}\right)+a\left(x_{0}\right) f_{1}\left(x_{0}\right)+c\left(x_{0}\right) f_{2}\left(x_{0}\right)-\gamma\left(x_{0}\right) f\left(x_{0}\right) \\
& +\int_{D_{x_{0}}}\left\{f\left(x_{0}+y\right)-f\left(x_{0}\right)-\xi_{1}(y) f_{1}\left(x_{0}\right)\right\} n_{x_{0}}(d y)+\sigma\left(x_{0}\right) A f\left(x_{0}\right)=0 .
\end{aligned}
$$

Furthermore if $\alpha^{2}\left(x_{0}\right)=a\left(x_{0}\right)=c\left(x_{0}\right)=\gamma\left(x_{0}\right)=\sigma\left(x_{0}\right)=0$ then $n_{x_{0}} \not \equiv 0$. We claim that $\sigma\left(x_{0}\right) \neq 0$. In fact, if $\sigma\left(x_{0}\right)=0$ then since $\left\{f_{\lambda} ; \lambda \gg 0\right\} \subset D(A) \cap C^{2}(V)$,

$$
\begin{aligned}
L f_{\lambda}\left(x_{0}\right)= & \left\{\lambda_{1}^{2} \alpha^{2}\left(x_{0}\right)-\lambda_{1} a\left(x_{0}\right)-\lambda_{2} c\left(x_{0}\right)-\gamma\left(x_{0}\right)\right. \\
& \left.+\int_{D_{x_{0}}}\left(e^{-\lambda \cdot y}-1-\lambda_{1} \xi_{1}(y)\right) n_{x_{0}}(d y)\right\} e^{-\lambda \cdot x_{0}}=0
\end{aligned}
$$

(11) $A f_{\lambda}(O)=0$ for every $\lambda \gg 0$.

${ }^{(12)} C_{0}^{\infty}\left(R^{n}\right)=\left\{f\right.$ : all derivatives of $\left.f \in C_{0}\left(R^{n}\right)\right\}$ where $C_{0}\left(R^{n}\right)=\left\{f\right.$ : continuous on $R^{n}$ and $\left.\lim _{|x| \rightarrow \infty}|f(x)|=0\right\}$. 
for all $\lambda \gg 0$. By a standard argument this implies that $\alpha^{2}\left(x_{0}\right)=a\left(x_{0}\right)=c\left(x_{0}\right)=\gamma\left(x_{0}\right)$ $=n_{x_{0}}(d y)=0$ and this is a contradiction. Hence $\sigma\left(x_{0}\right) \neq 0$. Without loss of the generality we may assume $\sigma\left(x_{0}\right)=-1$ then from $L f_{\lambda}\left(x_{0}\right)=0$ we have

$$
\begin{aligned}
A f_{\lambda}\left(x_{0}\right)=\left\{\lambda_{1}^{2} \alpha^{2}\left(x_{0}\right)-\lambda_{1} a\left(x_{0}\right)-\right. & \lambda_{1} c\left(x_{0}\right)-\gamma\left(x_{0}\right) \\
& \left.+\int_{D_{x_{0}}}\left(e^{-\lambda \cdot y}-1+\lambda_{1} \xi_{1}(y)\right) n_{x_{0}}(d y)\right\} e^{-\lambda \cdot x_{0}} .
\end{aligned}
$$

Set

$$
\begin{aligned}
h_{1}(\lambda)= & -e^{\lambda_{1}} A f_{\lambda}(1,0)=-\lambda_{1}^{2} \alpha^{2}+\left(a \lambda_{1}+c \lambda_{2}\right)+\gamma \\
& -\int_{D_{(1,0)}}\left(e^{-\lambda \cdot y}-1+\lambda_{1} \xi_{1}(y)\right) n_{1}(d y)
\end{aligned}
$$

where $\alpha^{2}=\alpha^{2}(1,0), a=a(1,0), \ldots, n_{1}(d y)=n_{(1,0)}(d y)$. We have a similar expression for $h_{2}(\lambda)=-e^{\lambda_{2}} A f_{\lambda}(0,1)$;

$$
\begin{aligned}
h_{2}(\lambda)= & -e^{\lambda_{2}} A f_{\lambda}(0,1)=-\lambda_{2} \beta^{2}+\left(b \lambda_{1}+d \lambda_{2}\right)+\delta \\
& -\int_{D_{(0,1)}}\left(e^{-\lambda \cdot y}-1+\lambda_{2} \xi_{2}(y)\right) n_{2}(d y) .
\end{aligned}
$$

By (2.14),

$$
A f_{\lambda}(x)=e^{-\lambda \cdot x}\left(-x_{1} h_{1}(\lambda)-x_{2} h_{2}(\lambda)\right)=\left(h_{1} \frac{\partial}{\partial \lambda_{1}}+h_{2} \frac{\partial}{\partial \lambda_{2}}\right) f_{\lambda}(x)
$$

If we set $x=x_{0}=\left(x_{1}^{0}, 0\right)$ in $(2.18)$ and compare this with (2.15) we see at once that

$$
\alpha^{2}\left(x_{0}\right)=x_{1}^{0} \alpha^{2}, \quad a\left(x_{0}\right)=x_{1}^{0} a, \ldots, n_{x_{0}}(d y)=x_{1}^{0} n_{1}(d y) .
$$

From the last relation, since $n_{x_{0}}(d y)$ is a measure on $D_{x_{0}}-\{O\}$, by letting $x_{0} \rightarrow 0$ we see at once that $n_{1}(d y)$ is a measure on $D-\{O\}$. Similarly $n_{2}(d y)$ is a measure on $D-\{O\}$. Thus we have proved that there exist $\alpha, \beta, \gamma, \delta, a, b, c, d, n_{1}$ and $n_{2}$ satisfying (1.10) and (1.11) such that if the operator $A^{\prime}$ is defined by (1.9) then

$$
A f_{\lambda}=A^{\prime} f_{\lambda} \quad \text { for all } \lambda \gg 0 .
$$

For any $f \in C_{0}^{2}(D)$ choose $f_{n} \in \mathscr{L}\left\{f_{\lambda} ; \lambda \gg 0\right\}\left({ }^{13}\right)$ such that $f_{n} \Rightarrow f, \partial f_{n} / \partial x_{i} \Rightarrow f$ and $\partial^{2} f_{n} /\left(\partial x_{i} \partial x_{j}\right) \Rightarrow \partial^{2} f /\left(\partial x_{i} \partial x_{j}\right) \quad(n \rightarrow \infty)$ then $A f_{n}=A^{\prime} f_{n} \rightarrow A^{\prime} f$ and hence by the closedness of the operator $A$, we have $C_{0}^{2}(D) \subset D(A)$ and for $f \in C_{0}^{2}(D), A f=A^{\prime} f$.

Since $f_{\lambda} \in D(A)$ we have

$$
\partial T_{t} f_{\lambda} / \partial t=A T_{t} f_{\lambda}=T_{t} A f_{\lambda}
$$

By (2.18),

$$
\frac{\partial T_{t} f_{\lambda}}{\partial t}=T_{t} A f_{\lambda}=T_{t}\left(h_{1} \frac{\partial f_{\lambda}}{\partial \lambda_{1}}+h_{2} \frac{\partial f_{\lambda}}{\partial \lambda_{2}}\right)=h_{1} \frac{\partial T_{t} f_{\lambda}}{\partial \lambda_{1}}+h_{2} \frac{\partial T_{t} f_{\lambda}}{\partial \lambda_{2}}
$$

(13) $\mathscr{L}\left\{f_{\lambda} ; \lambda \gg 0\right\}$ is the linear hull of $\left\{f_{\lambda} ; \lambda \gg 0\right\}$. 
and this proves $T_{t} f_{\lambda}(x)=F(t, \lambda ; x)$ is a solution of the forward equation (1.16). If we set $\exp \left(-\psi_{1}(t, \lambda)\right)=T_{t} f_{\lambda}(1,0)$ and $\exp \left(-\psi_{2}(t, \lambda)\right)=T_{t} f_{\lambda}(0,1)$ then for $i=1,2$,

$$
\begin{aligned}
\frac{d \psi_{i}(t, \lambda)}{d t} & =-\exp \left(\psi_{i}(t, \lambda)\right) \frac{\partial T_{t} f_{\lambda}}{\partial t}(1,0)=-\exp \left(\psi_{i}(t, \lambda)\right) A T_{t} f_{\lambda}(1,0) \\
& =-\exp \left(\psi_{i}(t, \lambda)\right) A\left(f_{\psi_{i}(t, \lambda)}\right)=h_{i}\left(\psi_{t}\right) \quad\left(\psi_{t}=\left(\psi_{1}(t, \lambda), \psi_{2}(t, \lambda)\right)\right)
\end{aligned}
$$

proving that $\psi_{t}$ is a solution of the backward equation (1.15).

Next we shall prove the existence of C.B.P. First we shall prove the uniqueness. Let $T_{t}$ and $\tilde{T}_{t}$ be strongly continuous semigroups on $C_{0}(D)$ with the infinitesimal generators $A$ and $\tilde{A}$ respectively such that $C_{0}^{2}(D) \subset D(A) \cap D(\tilde{A}), A=\tilde{A}$ on $C_{0}^{2}(D)$ and the expression of $A$ on $C_{0}^{2}(D)$ is given by (1.9). Then $T_{t} f_{\lambda}(x)$ and $\tilde{T}_{t} f_{\lambda}(x)$ satisfy the forward equation with the same initial value $e^{-\lambda \cdot x}$ and hence $F(t, \lambda)$ $=T_{t} f_{\lambda}(x)-\tilde{T}_{t} f_{\lambda}(x)$ satisfies

$$
\frac{\partial F}{\partial t}=h_{1}(\lambda) \frac{\partial F}{\partial \lambda_{1}}+h_{2}(\lambda) \frac{\partial F}{\partial \lambda_{2}}, \quad F(0+, \lambda)=0 .
$$

By Lemma 3, $F(t, \lambda) \equiv 0$, i.e., $T_{t} f_{\lambda}=\tilde{T}_{t} f_{\lambda}$ for every $\lambda \gg 0$. Since the linear hull $\left\{f_{\lambda} ; \lambda \gg 0\right\}$ is dense in $C_{0}(D)$ we have $T_{t}=\tilde{T}_{t}$.

Now we shall prove the existence. Given $\alpha, \beta, \gamma, \delta, a, b, c, d, n_{1}$ and $n_{2}$ satisfying the conditions (1.10) and (1.11) define $h_{1}(\lambda)$ and $h_{2}(\lambda)$ by (1.13). By the correspondence between C.B.P.'s and $\Psi^{2}$-semigroups established in Theorem 1 it is sufficient to prove that the solution $\psi_{t}(\lambda)$ of the backward equation:

$$
d \psi_{t} / d t=h\left(\psi_{t}\right), \quad \psi_{0+}=\lambda
$$

where $h=\left(h_{1}, h_{2}\right)$ defines a $\Psi^{2}$-semigroup. In fact it is easy to see that the C.B.P. corresponding to $\psi_{t}$ by Theorem 1 has the semigroup $T_{t}$ with the infinitesimal generator $A$ such that $\left\{f_{\lambda} ; \lambda \gg 0\right\} \subset D(A)$ and $A f_{\lambda}(x)$ is given by (2.18). Hence by the same argument as above $C_{0}^{2}(D) \subset D(A)$ and the expression of $A$ on $C_{0}^{2}(D)$ is given by (1.9). To show that $\Psi_{t}$ is a $\Psi^{2}$-semigroup it is clearly sufficient to prove that for some $t_{0}>0, \Psi_{t} \in \Psi^{2}, t \in\left[0, t_{0}\right]$ and $\Psi_{t+s}(\lambda)=\psi_{t}\left(\psi_{s}(\lambda)\right)$ for all $t, s \in\left[0, t_{0}\right]$ such that $t+s \in\left[0, t_{0}\right]$; then $\left\{\Psi_{t}\right\}_{t \in\left[0, t_{0}\right]}$ can be extended uniquely to a $\Psi^{2}$-semigroup $\left\{\boldsymbol{\Psi}_{t}\right\}_{t \in[0, \infty)}$. Since $h(\lambda)$ is Lipschitz continuous on every domain $U \Subset \Lambda=\{\lambda ; \lambda \geqq 0\}$, the solution of (1.15) exists uniquely on $U$ in $t \in\left[0, t_{0}\right]$ for some $t_{0}>0$. The semigroup property of $\psi_{t}$ is clear from the uniqueness of the solution. Hence what remains to be proved is that $\psi_{t} \in \Psi^{2}$ for all $t \in\left[0, t_{0}\right]$. For this we first consider the following simple case:

$$
\alpha^{2}=\beta^{2}=0, \quad n_{1}(D-\{O\})+n_{2}(D-\{O\})<\infty .
$$

Then it is easy to see that $h_{1}$ and $h_{2}$ can be represented in the form

$$
h_{i}(\lambda)=\sigma_{i}\left(\varphi_{i}(\lambda)-\lambda_{i}\right), \quad i=1,2,
$$

where $\sigma_{i}(i=1,2)$ are positive constants and $\varphi_{i} \in \Psi,(i=1,2)$. Then the equation 
(1.15) is equivalent to the following integral equation

$$
\psi_{i}(t, \lambda)=e^{-\sigma_{i} t} \lambda_{i}+\sigma_{i} \int_{0}^{t} e^{-\sigma_{i}(t-s)} \varphi_{i}\left(\psi_{s}\right) d s, \quad i=1,2 .
$$

Now if we define $\psi_{i}^{(n)}(\lambda)=\left(\psi_{1}^{(n)}(t, \lambda), \psi_{2}^{(n)}(t, \lambda)\right)$ successively by

$$
\begin{aligned}
& \psi_{i}^{(0)}(t, \lambda)=\lambda_{i}, \\
& \psi_{i}^{(n)}(t, \lambda)=e^{-\sigma_{i} t} \lambda_{i}+\sigma_{i} \int_{0}^{t} e^{-\sigma_{i}^{(t-s)}} \varphi_{i}\left(\psi_{s}^{(n-1)}\right) d s, \quad i=1,2,
\end{aligned}
$$

then noting that $\Psi^{2}$ is closed under the composition we see easily by induction that $\psi_{t}^{(n)} \in \Psi^{2}$. Hence by Lemma $1, \Psi_{t} \in \Psi^{2}$ as the uniform limit of $\psi_{t}^{(n)}$ on a domain $U \Subset \Lambda$. To prove the general case we remark first that given $h=\left(h_{1}, h_{2}\right)$ there exists a sequence $h_{n}=\left(h_{1}^{(n)}, h_{2}^{(n)}\right)$ such that for each $n, h_{n}$ has the property (2.19) and $h_{n} \Rightarrow h$ on every $U \subset \Lambda$. This is a well known fact and can be proved in a similar method as the proof of Lemma 1 . Let $\psi_{t}^{(n)}$ be the solution of (1.15) corresponding to $h_{n}$ then $\Psi_{t}^{(n)} \in \Psi^{2}$ and by Lemma $2, \Psi_{t}^{(n)} \Rightarrow \psi_{t}$ on some $U_{0} \Subset \Lambda$ and $t \in\left[0, t_{0}\right]$. Hence by Lemma $1, \Psi_{t} \in \Psi^{2}$ and the proof of Theorem 2 is now complete.

Consider the C.B.P. $X=\left(x_{t}, P_{x}\right)$ of Theorem 2 . Since its semigroup is strongly continuous on $C_{0}(D), X$ is a standard process or Hunt process, cf. Dynkin [1]. In particular we may assume that it is a strong Markov process with right continuous and $d_{1}$-discontinuous sample functions. It is a diffusion process, i.e., its sample functions are continuous with probability one if and only if $\gamma=\delta=n_{1}$ $=n_{2}=0$. A probabilistic meaning of $\gamma, \delta, n_{1}, n_{2}$ is the following. Define a nonnegative kernel $n(x, d y)$ on $D \times \bar{D}$ by

$$
\begin{array}{r}
n(x, E)=x_{1} n_{1}\left(E_{x} \cap D\right)+x_{2} n_{2}\left(E_{x} \cap D\right)+\left(\gamma x_{1}+\delta x_{2}\right) \delta_{\Delta}(E), \\
E \in \mathscr{B}(\bar{D}), x=\left(x_{1}, x_{2}\right)\left({ }^{14}\right) .
\end{array}
$$

Let $f(x, y)$ be any nonnegative function on $\bar{D} \times \bar{D}$ such that $f(x, x)=0$ for all $x \in \bar{D}$. Then for every $x \in D$ and $t \geqq 0$ we have

$$
E_{x}\left[\sum_{s \leqq t} f\left(x_{s-}, x_{s}\right)\right]=E\left[\int_{0}^{t}\left\{\int_{\bar{D}} f\left(x_{s}, y\right) n\left(x_{s}, d y\right)\right\} d s\right]
$$

where $x_{s-}$ is the left hand limit of $x_{t}$ at $s$ (cf. Watanabe [16]).

3. The case of diffusion processes. In this section we shall study the case of diffusion processes, i.e., the case $\gamma=\delta=n_{1}=n_{2}=0$. The infinitesimal generator $A$ given in Theorem 2 has now the following form:

$$
A f=\alpha^{2} x_{1} f_{11}+\beta^{2} x_{2} f_{22}+\left(a x_{1}+b x_{2}\right) f_{1}+\left(c x_{1}+d x_{2}\right) f_{2} .
$$

${ }^{(14)} E_{x}=\{y-x ; y \in E\}, \mathscr{B}(\bar{D})$ is the set of all Borel subsets of $D^{*}$ and $\delta_{\Delta}(E)$ is the unit measure on the point at infinity $\Delta$. 
If $b=c=0$ then the corresponding C.B.P. is the direct product of two onedimensional Feller diffusions. This class of 2-dimensional diffusions was discussed by Feller [2] in connection with Galton-Watson processes of two types.

The behavior of sample functions on $x_{1}$ axis when $c=0$ (or on $x_{2}$-axis when $b=0)$ is the following. If a sample path reaches the $x_{1}$-axis then it remains on $x_{1}$ axis moving as a one-dimensional Feller diffusion determined by the infinitesimal generator $\alpha^{2} x\left(d^{2} / d x^{2}\right)+a x(d / d x)$. Finally it hits the origin $O$ and then it is stopped. The probability that a sample function hits the $x_{1}$-axis is positive if $\beta \neq 0$.

From now on we shall consider the case when $b>0$ and $c>0$. Assume for simplicity that $\alpha=\beta=1$. It is natural to expect that the process $X=\left(x_{t}, P_{x}\right)$ is obtained as a solution of the following stochastic equation;

$$
\begin{aligned}
& d x_{1}(t)=\left(x_{1}(t)^{+}\right)^{1 / 2} d B_{t}^{(1)}+\left(a x_{1}(t)+b x_{2}(t)\right) d t\left({ }^{15}\right), \\
& d x_{2}(t)=\left(x_{2}(t)^{+}\right)^{1 / 2} d B_{t}^{(2)}+\left(c x_{1}(t)+d x_{2}(t)\right) d t
\end{aligned}
$$

where $B_{t}^{(1)}$ and $B_{t}^{(2)}$ are mutually independent Wiener processes. The coefficients are Lipschitz continuous on every $U \Subset D$ and hence by Ito's theorem (cf. [6], [13]) the solution of (3.2) exists uniquely up to the first hitting time for the boundary. Since the coefficients are not Lipschitz continuous we cannot apply Ito's theorem for existence and uniqueness of a global solution of (3.2) but we have the following

THEOREM 3. For any $x \in D$, there exists a unique solution $x_{t}=\left(x_{1}(t), x_{2}(t)\right)$ of the equation (3.2) with $x_{0}=x$ such that it is stopped at the origin after it hits there for the first time. The solution defines a diffusion process $X=\left(x_{t}, P_{x}\right)$ and this coincides with the C.B.P. given in Theorem 2.

Proof. Since the coefficients are continuous on the whole plane there exists a solution of (3.2) for any initial value $x_{0}=x \in R^{n}$ by Skorohod's existence theorem $[13$, p. 59]. We modify this solution so that it is stopped at the origin after it hits there for the first time. This gives also a solution of (3.2). If the initial point $x$ is in $D$ then this solution $x_{t}$ never leaves $D$. In fact, if it leaves $D$, there exists some time interval $\left[t_{0}, t_{1}\right]$ such that $x_{t_{0}} \in \partial D$, say on $x_{1}$-axis (i.e., $\left.x_{2}\left(t_{0}\right)=0\right)$ and $x_{2}(t)<0$ for $t \in\left[t_{0}, t_{1}\right]$. But on a neighborhood of $x_{t_{0}}, c x_{1}+d x_{2} \geqq \varepsilon>0$ for some positive constant $\varepsilon$ and hence $x_{2}(t)-x_{2}(s)=\int_{s}^{t}\left[c x_{1}(u)+d x_{2}(u)\right] d u \geqq \varepsilon(t-s)$ for every $s, t$ $\in\left[t_{0}, t\right]$. This implies that $x_{2}(t) \geqq x_{2}\left(t_{0}\right)+\varepsilon\left(t-t_{0}\right)$ and contradicts with $x_{2}(t)<0$ for $t \in\left[t_{0}, t_{1}\right]$.

Now we shall show the uniqueness of such solutions. It is sufficient to show the uniqueness of the solution on a neighborhood of each boundary point since then the uniqueness of the global solution follows from the usual piecing out method. Let $x^{(0)}=\left(x_{1}^{0}, 0\right), x_{1}^{0}>0$ and $U$ be a sufficiently small neighborhood of $x_{0}$ such that $c x_{1}+d x_{2}>\varepsilon>0$ on $U$ where $\varepsilon$ is a positive constant. Let $x_{t}$ and $\tilde{x}_{t}$ be two solutions such that $x_{0}=\tilde{x}_{0}=x^{(0)}$. We shall now prove $x_{t}=\tilde{x}_{t}$ for $t<\tau_{U}$ where $\tau_{U}$ is the first leaving time from $U$. Let $A(x), B(x), C(x)$ and $D(x)$ be bounded continuous

(15) $x_{t}=\left(x_{1}(t), x_{2}(t)\right), \alpha^{+}=\alpha \vee 0$. 
functions on $R^{2}$ such that

(i) $A(x) \equiv A\left(x_{1}\right)$; a function of $x_{1}$ only and $A\left(x_{1}\right)=\left(x_{1}^{+}\right)^{1 / 2}$ on $U$,

(ii) $B(x)=B\left(x_{2}\right)$; a function of $x_{2}$ only and $B\left(x_{2}\right)=\left(x_{2}^{+}\right)^{1 / 2}$ on $U$ and $B\left(x_{2}\right)$ $\leqq\left(2 x_{2}^{+}\right)^{1 / 2}$, everywhere,

(iii) $C(x)=a x_{1}+b x_{2}$ on $U$,

(iv) $D(x)=c x_{1}+d x_{2}$ on $U$ and $D(x) \geqq \varepsilon$ everywhere,

(v) $A, C, D$ are Lipschitz continuous and $B(x)$ is Lipschitz continuous on $\left|x_{2}\right|>k>0$ for every $k>0$.

Clearly such $A, B, C$ and $D$ exist. In order to show that $x_{t}=\tilde{x}_{t}$ for $t<\tau_{U}$ it is sufficient to prove that, if $y_{t}$ and $\tilde{y}_{t}$ are two solutions of

$$
\begin{aligned}
& d x_{1}(t)=A\left(x_{t}\right) d B_{t}^{(1)}+C\left(x_{t}\right) d t, \\
& d x_{2}(t)=B\left(x_{t}\right) d B_{t}^{(2)}+D\left(x_{t}\right) d t
\end{aligned}
$$

such that $y_{0}=\tilde{y}_{0}=x^{(0)}$, then $y_{t} \equiv \tilde{y}_{t}$. First we remark that by the same argument as above we can prove that any solution $y_{t}=\left(y_{1}(t), y_{2}(t)\right)$ of (3.3) with $y_{0}=x^{(0)}$ satisfies $y_{2}(t) \geqq 0$ for all $t \geqq 0$.

LEMMA 4. There exists $\alpha, 0<\alpha<1$ such that if $y_{t}=\left(y_{1}(t), y_{2}(t)\right)$ is a solution of (3.3) with $y_{0}=x^{(0)}$ then

$$
\int_{0}^{t} E\left\{y_{2}(s)^{\alpha-1}\right\} d s \quad\left(=E\left\{\int_{0}^{t} y_{2}(s)^{\alpha-1} d s\right\}\right)<\infty .
$$

Proof. Let $\eta>0$; then by a formula on stochastic integrals due to Ito (cf. [6], [13])

$$
\begin{aligned}
\left(y_{2}(t)+\eta\right)^{\alpha}-\eta^{\alpha}= & \alpha \int_{0}^{t}\left(y_{2}(s)+\eta\right)^{\alpha-1} B\left(y_{s}\right) d B_{s}^{(2)}+\alpha \int_{0}^{t}\left(y_{2}(s)+\eta\right)^{\alpha-1} D\left(y_{s}\right) d s \\
& +\frac{\alpha(\alpha-1)}{2} \int_{0}^{t}\left(y_{2}(s)+\eta\right)^{\alpha-2} B^{2}\left(y_{s}\right) d s .
\end{aligned}
$$

By taking the expectations of both sides we have

$$
\begin{aligned}
E\left\{\left(y_{2}(t)+\eta\right)^{\alpha}\right\}-\eta^{\alpha}= & \alpha \int_{0}^{t} E\left\{\left(y_{2}(s)+\eta\right)^{\alpha-1} D\left(y_{s}\right)\right\} d s \\
& +\frac{\alpha(\alpha-1)}{2} \int_{0}^{t} E\left\{\left(y_{2}(s)+\eta\right)^{\alpha-2} B^{2}\left(y_{s}\right)\right\} d s .
\end{aligned}
$$

By the properties of $B$ and $D, B^{2}(y) \leqq 2 y_{2}^{+}$and $D \geqq \varepsilon$ and hence if $1-\varepsilon<\alpha<1$ then

$$
\begin{aligned}
E\left\{\left(y_{2}(t)+\eta\right)^{\alpha}\right\}-\eta^{\alpha} \geqq & \alpha \varepsilon \int_{0}^{t} E\left\{\left(y_{2}(s)+\eta\right)^{\alpha-1}\right\} d s \\
& -\alpha(1-\alpha) \int_{0}^{t} E\left\{\left(y_{2}(s)+\eta\right)^{\alpha-2} y_{2}(s)\right\} d s \\
\geqq & \alpha\{\varepsilon-(1-\alpha)\} \int_{0}^{t} E\left\{\left(y_{2}(s)+\eta\right)^{\alpha-1}\right) d s
\end{aligned}
$$

Letting $\eta \downarrow 0$ we have

$$
\infty>E\left\{y_{2}(t)^{\alpha}\right\} \geqq \alpha\{\varepsilon-(1-\alpha)\} \int_{0}^{t} E\left\{y_{2}(s)^{\alpha-1}\right\} d s
$$

proving (3.4). 
Now we shall prove $y_{t}=\tilde{y}_{t}$. The following argument was suggested by a beautiful proof of Tanaka [14] for the uniqueness of solutions with coefficients Hölder continuous of exponent $>1 / 2$. Let $f_{n}(\alpha), \alpha \in R^{1}$, be defined by

$$
\begin{aligned}
f_{n}(\alpha) & =|\alpha|, & & |\alpha|>1 / n, \\
& =\left(1+n^{2} \alpha^{2}\right) / 2 n, & & |\alpha| \leqq 1 / n .
\end{aligned}
$$

By Ito's formula on stochastic integrals we have

$$
\begin{aligned}
& f_{n}\left(y_{2}(t)-\tilde{y}_{2}(t)\right) \\
&=\int_{0}^{t} f_{n}^{\prime}\left(y_{2}(s)-\tilde{y}_{2}(s)\right)\left(B\left(y_{s}\right)-\left(B \tilde{y}_{s}\right)\right) d B_{s}^{(2)}+\int_{0}^{t} f_{n}^{\prime}\left(y_{2}(s)-\tilde{y}_{2}(s)\right) \\
& \times\left(D\left(y_{s}\right)-D\left(\tilde{y}_{s}\right)\right) d s+\frac{1}{2} \int_{0}^{t} f_{n}^{\prime \prime}\left(y_{2}(s)-\tilde{y}_{2}(s)\right)\left(B\left(y_{s}\right)-B\left(\tilde{y}_{s}\right)\right)^{2} d s \\
&= I_{1}+I_{2}+I_{3}, \quad \text { say. }
\end{aligned}
$$

Then $\left({ }^{16}\right)$

$$
\begin{aligned}
E\left\{f_{n}\left(y_{2}(t)-\tilde{y}_{2}(t)\right)\right\} & \rightarrow E\left|y_{2}(t)-\tilde{y}_{2}(t)\right|, \quad(n \rightarrow \infty) \\
E\left\{I_{1}\right\} & =0 \quad \text { for every } n,
\end{aligned}
$$

and

$$
E\left\{I_{2}\right\} \leqq K_{1} \int_{0}^{t} E\left\{\left|y_{1}(s)-\tilde{y}_{1}(s)\right|+\left|y_{2}(s)-\tilde{y}_{2}(s)\right|\right\} d s
$$

since $f_{n}^{\prime}(\alpha)$ is uniformly bounded and $D(y)$ is Lipschitz continuous. If $y, y^{\prime} \in D$

$$
\left|B(y)-B\left(y^{\prime}\right)\right|^{2} \leqq K_{2}\left|y_{2}^{1 / 2}-y_{2}^{\prime 1 / 2}\right|^{2} \leqq K_{3}\left|y_{2}-y_{2}^{\prime}\right|^{2-\alpha} y_{2}^{\alpha-1}
$$

for every $\alpha(0<\alpha<1)$. Then

$$
\begin{aligned}
I_{3} & =\frac{1}{2} \int_{0}^{t} f_{n}^{\prime \prime}\left(y_{2}(s)-\tilde{y}_{2}(s)\right)\left(B\left(y_{s}\right)-B\left(\tilde{y}_{s}\right)\right)^{2} d s \\
& =\frac{n}{2} \int_{0}^{t} I\left\{\left|y_{2}(s)-\tilde{y}_{2}(s)\right| \leqq \frac{1}{n}\right\}\left(B\left(y_{s}\right)-B\left(\tilde{y}_{s}\right)\right)^{2} d s\left({ }^{17}\right) \\
& \leqq K_{3} \frac{n}{2} n^{\alpha-2} \int_{0}^{t} y_{2}(s)^{\alpha-1} d s
\end{aligned}
$$

and hence, if we choose $\alpha$ so that (3.4) holds, $E\left\{I_{3}\right\} \rightarrow 0$ when $n \rightarrow \infty$. Therefore

$$
E\left\{\left|y_{2}(t)-\tilde{y}_{2}(t)\right|\right\} \leqq K_{1} \int_{0}^{t} E\left\{\left|y_{1}(s)-\tilde{y}_{1}(s)\right|+\left|y_{2}(s)-\tilde{y}_{2}(s)\right|\right\} d s .
$$

By a similar argument we can prove

$$
E\left\{\left|y_{1}(t)-\tilde{y}_{1}(t)\right|\right\} \leqq K_{4} \int_{0}^{t} E\left\{\left|y_{1}(s)-\tilde{y}_{1}(s)\right|+\left|y_{2}(s)-\tilde{y}_{2}(s)\right|\right\} d s .
$$

${ }^{\left({ }^{16}\right)}$ In the following $K_{1}, K_{2}, \ldots$ are positive constants.

(17) $I_{E}$ denotes the indicator function of an event $E$. 
In fact,

$$
\begin{aligned}
I_{3}^{\prime} & \equiv \frac{1}{2} \int_{0}^{t} f_{n}^{n}\left(y_{1}(s)-\tilde{y}_{1}(s)\right)\left(A\left(y_{s}\right)-A\left(\tilde{y}_{s}\right)\right)^{2} d s \\
& =\frac{n}{2} \int_{0}^{t} I\left\{\left|y_{1}(s)-\tilde{y}_{1}(s)\right| \leqq \frac{1}{n}\right\}\left(A\left(y_{s}\right)-A\left(\tilde{y}_{s}\right)\right)^{2} d s \\
& \leqq \frac{K_{5}}{2} n \int_{0}^{t} I\left\{\left|y_{1}(s)-\tilde{y}_{1}(s)\right| \leqq \frac{1}{n}\right\}\left(y_{1}(s)-\tilde{y}_{1}(s)\right)^{2} d s \\
& \leqq \frac{K_{5}}{2} n \cdot n^{-2} t \rightarrow 0, \quad(n \rightarrow \infty)
\end{aligned}
$$

by the Lipschitz continuity of $A$ and hence we get (3.8) just as above. From (3.7) and (3.8), if we set

$$
A(t)=E\left\{\left|y_{1}(t)-\tilde{y}_{1}(t)\right|+\left|y_{2}(t)-\tilde{y}_{2}(t)\right|\right\}
$$

then we have

$$
A(t) \leqq K_{6} \int_{0}^{t} A(s) d s
$$

and hence $A(t) \equiv 0$ by a usual argument. Thus $y_{t} \equiv \tilde{y}_{t}$.

Finally we shall show that the diffusion process $X=\left(x_{t}, P_{x}\right)$ defined by the above unique solution of (3.2) coincides with the C.B.P. of Theorem 2. Again by Ito's formula we have for every $\lambda \gg 0$,

$$
\begin{aligned}
e^{-\lambda \cdot x_{t}}-e^{-\lambda \cdot x_{0}}= & \lambda_{1} \int_{0}^{t} x_{1}(s)^{1 / 2} e^{-\lambda \cdot x_{s}} d B_{s}^{(1)}+\lambda_{2} \int_{0}^{t} x_{2}(s)^{1 / 2} e^{-\lambda \cdot x_{s}} d B_{s}^{(2)} \\
& +\frac{\lambda_{1}^{2}}{2} \int_{0}^{t} x_{1}(s) e^{-\lambda \cdot x_{s}} d s+\frac{\lambda_{2}^{2}}{2} \int_{0}^{t} x_{2}(s) e^{-\lambda \cdot x_{s}} d s \\
& -\lambda_{1} \int_{0}^{t}\left(a x_{1}(s)+b x_{2}(s)\right) e^{-\lambda \cdot x_{s}} d s \\
& -\lambda_{2} \int_{0}^{t}\left(c x_{1}(s)+d x_{2}(s)\right) e^{-\lambda \cdot x_{s}} d s
\end{aligned}
$$

and hence by taking the expectation of both sides

$$
\begin{aligned}
E_{x}\left(e^{-\lambda \cdot x_{t}}\right) & =e^{-\lambda \cdot x}-h_{1}(\lambda) E_{x}\left\{\int_{0}^{t} x_{1}(s) e^{-\lambda \cdot x_{s}} d s\right\}-h_{2}(\lambda) E_{x}\left\{\int_{0}^{t} x_{2}(s) e^{-\lambda \cdot x_{s}} d s\right\} \\
& =e^{-\lambda \cdot x}+\int_{0}^{t}\left[\left(h_{1} \frac{\partial}{\partial \lambda_{1}}+h_{2} \frac{\partial}{\partial \lambda_{2}}\right) E_{x}\left(e^{-\lambda \cdot x_{s}}\right)\right] d s .
\end{aligned}
$$

Hence $F(t, \lambda ; x)=E_{x}\left(e^{-\lambda \cdot x_{t}}\right)$ satisfies the forward equation

$$
\frac{\partial F}{\partial t}=h_{1}(\lambda) \frac{\partial F}{\partial \lambda_{1}}+h_{2}(\lambda) \frac{\partial F}{\partial \lambda_{2}}, \quad F(0+, \lambda ; x)=e^{-\lambda \cdot x} .
$$

By Lemma 3, $X$ coincides with C.B.P. of Theorem 2. 
REMARK. The branching property of $X=\left(x_{t}, P_{x}\right)$ can be seen directly in the following way: we have shown above that $F(t, \lambda ; x)=E_{x}\left(e^{-\lambda \cdot x_{t}}\right)$ is the solution of the forward equation (1.16). On the other hand, as is easily seen, $\tilde{F}(t, \lambda ; x)$ $=E_{(1,0)}\left(e^{-\lambda \cdot x_{t}}\right)^{x_{1}} E_{(0,1)}\left(e^{-\lambda \cdot x_{t}}\right)^{x_{2}}$ satisfies (1.16). Hence by the uniqueness of the solution of the forward equation (Lemma 3 ) we have $\tilde{F}(t, \lambda ; x)=F(t, \lambda ; x)$ i.e., $X$ has the branching property.

From Lemma 4 we have at once the following

Corollary. If $\alpha=\beta=1, b>0$ and $c>0$ then the diffusion $X=\left(x_{t}, P_{x}\right)$ has the following property; with probability one the Lebesgue measure of the set of all $t$ such that $x_{t} \in \partial D-\{O\}$ ( $x_{1}$-axis or $x_{2}$-axis except origin) is 0 .

This fact is in striking contrast to the case when $b=0$ or $c=0$. We shall study in more detail the behavior of sample functions near the boundary. Set

$$
\begin{aligned}
& \Sigma_{1}=\left\{x=\left(x_{1}, 0\right) ; 1 / c<x_{1}<\infty\right\} \\
& \Sigma_{2}=\left\{x=\left(x_{1}, 0\right) ; 0<x_{1}<1 / c\right\} \\
& \Sigma_{3}=\left\{x=\left(0, x_{2}\right) ; 0<x_{2}<1 / b\right\}
\end{aligned}
$$

and

$$
\Sigma_{4}=\left\{x=\left(0, x_{2}\right) ; x_{2}>1 / b\right\} .
$$

Let $\bar{x} \in \partial D-\{O\}$ and $U$ be a neighborhood in $D$ of $\bar{x}$ which has the strictly positive distance from the origin. For simplicity we assume that $\bar{x}$ is on $x_{1}$-axis. For each $\eta>0$ set

$$
U^{\eta}=U \cap\left\{x=\left(x_{1}, x_{2}\right) ; x_{2}>\eta\right\}
$$

and let $\tau_{\eta}$ be the first leaving time from $U^{\eta}$. Set $\sigma=\lim _{\eta \downarrow} \tau_{\eta}$ and

$$
\Gamma_{U}^{\sigma}=\text { the (random) set of all limit points of } x_{t} \text { when } t \uparrow \sigma .
$$

Following Has'minsky [4], we shall give the following

Definition 1.5. $\bar{x}$ is called regular if for every neighborhood $V$ in $D$ of $\bar{x}$ we have

$$
\lim _{x \in D^{\circ} \rightarrow \bar{x}} P_{x}\left\{\Gamma_{U}^{\sigma} \subset V \cap \partial D\right\}=1
$$

where $U$ is a fixed neighborhood of $\bar{x}$.

Definition 1.6. Let $\Sigma$ be an open interval in $\partial D-\{O\} . \Sigma$ is called unattainable if for every $\bar{x} \in \Sigma$ there exists a neighborhood $U$ in $D$ of $\bar{x}$ such that

$$
P_{x}\left(\Gamma_{U}^{\sigma} \cap \partial D=\varnothing\right)=1, \quad \text { for every } x \in U \cap D^{\circ} .
$$

It is clear from these definitions that

(i) if $\Sigma$ is unattainable then $\Sigma$ consists of all irregular points, 
(ii) $\Sigma$ is unattainable if and only if each $x \in \Sigma$ has a neighborhood in $\Sigma$ which is unattainable,

(iii) if $\Sigma$ is unattainable then with probability one sample functions starting from a point in $D^{\circ}$ never hit $\Sigma$.

THEOREM 4. (i) $\Sigma_{2}$ and $\Sigma_{3}$ consist of all regular points. Furthermore sample functions hit any nonempty open set in $\Sigma_{2} \cup \Sigma_{3}$ in a finite time with positive probability.

(ii) $\Sigma_{1}$ and $\Sigma_{4}$ are unattainable.

Proof. Let $\bar{x}=\left(\bar{x}_{1}, 0\right) \in \Sigma_{2}$, i.e., $0<c \bar{x}_{1}<1$ and $I$ be a neighborhood in $\Sigma_{2}$ of $\bar{x}$. According to [4] set

$$
A_{*}\left(x_{2}\right)=\min _{x_{1} \in I} \frac{x_{2}}{x_{1}+\left|a x_{1}+b x_{2}\right|}, \quad B^{*}\left(x_{2}\right)=\max _{x_{1} \in I} \frac{c x_{1}+d x_{2}}{x_{2}}
$$

then it is easy to see that, if $I$ is sufficiently small, then there exist constants $0<k_{1}<k_{2}, 0<k_{3}<k_{4}<1$ such that $k_{1} x_{2} \leqq A^{*}\left(x_{2}\right) \leqq k_{2} x_{2}$ and $k_{3} / x_{2} \leqq B^{*}\left(x_{2}\right) \leqq k_{4} / x_{2}$. Then

$$
Z^{*}\left(x_{2}\right)=\exp \left\{\int_{x_{2}} B^{*}(z) d z\right\} \cdot \int_{x_{2}}\left[\exp \left\{\int_{z} B^{*}(u) d u\right\} \cdot A_{*}(z)\right]^{-1} d z \leqq k_{5} x_{2}^{-k_{4}}
$$

and hence $\int_{0+} Z^{*}\left(x_{2}\right) d x_{2}<\infty$. By Theorem 3.1 of [4] $\bar{x}$ is regular. Next we shall show that sample functions hit every nonempty open subset of $\Sigma_{2}$ with positive probability. Let $\bar{x} \in \Sigma_{2}$ and $U$ be a neighborhood in $D$ of $\bar{x}$ such that $c x_{1}+d x_{2}$ $\geqq \varepsilon>0$ on $U$ where $\varepsilon$ is a positive constant. Let $u(x) \in C_{0}^{2}(D)$ such that $u(x)=x_{2}$ on $U$ then $u(x) \in D(A)$ by Theorem 2 and $A u(x)=c x_{1}+d x_{2} \geqq \varepsilon$ on $U$. Let $\sigma$ be the first leaving time from $U \cap D^{\circ}$ and let $\sigma^{n}=\sigma \wedge n$. By Dynkin's formula [1] we have for any $x \in U \cap D^{\circ}$

$$
E_{x}\left(u\left(x_{\sigma_{n}}\right)\right)-u(x)=E_{x}\left[\int_{0}^{\sigma_{n}} A u\left(x_{s}\right) d s\right] \geqq \varepsilon E_{x}\left[\sigma^{n}\right]
$$

and letting $n \rightarrow \infty, E_{x}(\sigma) \leqq(2 / \varepsilon)\|u\|<\infty$. Hence $P_{x}[\sigma<\infty]=1$. If $P_{x}\left[x_{\sigma} \in \partial D\right]=0$, $\left.x \in U \cap D^{\circ}{ }^{18}\right)$, then every point of $U \cap \partial D$ would be irregular and this contradicts with what we have already proved. Hence $P_{x}\left[x_{\sigma} \in \partial D\right]>0$ for every $x \in D^{\circ} \cup U$.

Finally we shall show that $\Sigma_{1} \cup \Sigma_{4}$ is unattainable. Let $\bar{x}=\left(\bar{x}_{1}, 0\right) \in \Sigma_{1}$, i.e., $c \bar{x}_{1}>1$. Let $I$ be a neighborhood in $\Sigma_{1}$ of $\bar{x}$ and set

$$
A^{*}\left(x_{2}\right)=\max _{x_{1} \in I} \frac{x_{2}}{x_{1}+\left|a x_{1}+b x_{2}\right|}, \quad B_{*}\left(x_{2}\right)=\min _{x_{1} \in I} \frac{c x_{1}+d x_{2}}{x_{2}} .
$$

If $I$ is sufficiently small then there exist constants $k_{1}>0,1<k_{2}<k_{3}$ such that

${ }^{(18)}$ Note that if $P_{x}\left[x_{\sigma} \in \partial D\right]=0$ for at least one point $x \in D^{\circ} \cup U$ then $P_{x}\left[x_{\sigma} \in \partial D\right] \equiv 0$ on $D^{\circ} \cup U$ since it is an $A$-harmonic function. 
$A^{*}\left(x_{2}\right) \leqq k_{1} x_{2}$ and $k_{2} / x_{2} \leqq B^{*}\left(x_{2}\right) \leqq k_{3} / x_{2}$. Then

$$
Z_{*}\left(x_{2}\right)=\exp \left\{\int_{x_{2}} B_{*}(z) d z\right\} \cdot \int_{x_{2}}\left[\exp \left\{\int_{z} B_{*}(u) d u\right\} A^{*}(z)\right]^{-1} d z \geqq k_{4} x_{2}^{-k_{2}}
$$

and hence $\int_{0+} z\left(x_{2}\right) d x_{2}=+\infty$. By Theorem 3.2 of [4] $\bar{x}$ has a neighborhood in $\Sigma_{2}$ which is unattainable.

Irregularity of a point $\Sigma_{1}$ (or $\Sigma_{4}$ ) is seen more directly in the following way. Let $\bar{x}=\left(\bar{x}_{1}, 0\right)$ such that $c \bar{x}_{1}>1$ and let $U$ be a neighborhood of $\bar{x}$ such that it surrounded by a segment $\left[\sigma_{1}, \sigma_{2}\right]$ on $x_{1}$-axis $\left(1 / c<\sigma_{1}<\bar{x}_{1}<\sigma_{2}\right)$ and a smooth curve $\gamma$ in $D^{\circ}$ joining the points $\left(\sigma_{1}, 0\right)$ and $\left(\sigma_{2}, 0\right)$. If $k>0$ is a constant and $u \in C^{2}(U)$ such that $u \mid \gamma=0$ then by Green's formula we have easily that

$$
\begin{aligned}
\iint_{U}(A-k) u \cdot u d x= & -\iint_{U}\left\{x_{1} u_{1}^{2}+x_{2} u_{2}^{2}\right\} d x-\left(k+\frac{b}{2}+\frac{d}{2}\right) \iint_{U} u^{2} d x \\
& -\frac{1}{2} \int_{\sigma_{1}}^{\sigma_{2}}\left(c x_{1}-1\right) u^{2}\left(x_{1}, 0\right) d x_{1} .
\end{aligned}
$$

Hence if $k>-b / 2-\alpha / 2$ and $(A-k) u=0$ then $u \equiv 0$ in $U$. This shows that any solution of $(A-k) u=0$ is uniquely determined by the boundary value on the part $\gamma$ of the boundary and hence every point $x \in U \cap \partial D$ must be irregular points $\left({ }^{19}\right)$.

By Corollary of Theorem 3 the Lebesgue measure of the set of all $t$ such that $x_{t} \in \partial D-\{O\}$ is zero with probability one. Let $\mathfrak{M}\left({ }^{20}\right)$ be the set of all additive functionals which are square-integrable martingales. Then by a result of [12] $\mathfrak{M}$ is generated by

$$
M_{1}(t)=\int_{0}^{t} x_{1}(t)^{1 / 2} d B_{t}^{(1)} \quad \text { and } \quad M_{2}(t)=\int_{0}^{t} x_{2}(t)^{1 / 2} d B_{t}^{(2)} .
$$

Hence for every $M \in \mathfrak{M}, \int_{0}^{t} I_{\partial D}\left(x_{s}\right) d M_{s}=0$. Generally it is natural to call a part $\Sigma$ of the boundary a reflecting barrier if $\left({ }^{21}\right)$

(i) it consists of all regular points,

(ii) the sample functions hit every nonempty open subset of $\Sigma$ with positive probability,

(iii) the set of time $t$ such that $x_{t} \in \Sigma$ has the Lebesgue measure 0 with probability one,

(iv) for every $M \in \mathfrak{M}, \int_{0}^{t} I_{\Sigma}\left(x_{s}\right) d M_{s}=0$.

Thus we have the following

COROllary 1. $\Sigma=\Sigma_{1} \cup \Sigma_{2}$ is a reflecting barrier, i.e., it satisfies the conditions (i)-(iv).

$\left.{ }^{(19}\right)$ By the classification of Fichera [3], $\Sigma_{1} \cup \Sigma_{4}$ is $\Sigma^{(1)}$-boundary and $\Sigma_{2} \cup \Sigma_{3}$ is $\Sigma^{(2)}$. boundary.

$\left({ }^{20}\right)$ This class of additive functionals was studied in Motoo-Watanabe [12].

$\left.{ }^{21}\right)$ In the case of one-dimensional diffusion these conditions characterize a reflecting barrier among all possible boundary conditions, cf. Ito and McKean [7]. 
Also it is natural to call a part $\Sigma$ of the boundary a pure entrance boundary if $\Sigma$ is unattainable and every $\bar{x} \in \Sigma$ has a neighborhood $U$ such that if $\tau_{U}$ is the first leaving time from $U$ then $P_{x}\left(\tau_{U}<\infty\right)=1$ for every $x \in U$. For any $\bar{x} \in \Sigma_{1} \cup \Sigma_{4}$ we can show the existence of such a neighborhood $U$ in $D$ of $\bar{x}$ by the same argument as above involving Dynkin's formula. Thus we have the following

COROLlaRy 2. $\Sigma=\Sigma_{1} \cup \Sigma_{4}$ is a pure entrance boundary.

4. A remark on the regularity. The regularity of a C.B.P. or equivalently the regularity of a $\Psi^{2}$-semigroup follows from a much weaker condition as we shall see in the following

Theorem 5. A C.B.P. $X=\left\{x_{t}, P_{x}\right\}$ is regular if $\Psi_{t}(\lambda)$ is continuous in $t$ for each $\lambda \gg 0$.

REMARK. As Lamperti showed in Lemma 2.3 of [11] the continuity follows from the measurability and some additional condition.

Proof. From the continuity of $\psi_{t}(\lambda)$ we can conclude easily that $X$ is a Hunt process. Next we shall remark that there exist $u_{1}(x), u_{2}(x) \in D(A) \cap C^{\infty}(D)$ such that

$$
e^{-\lambda \cdot x}=\varphi^{(\lambda)}(u(x)), \quad \boldsymbol{u}(x)=\left(u_{1}(x), u_{2}(x)\right),
$$

where $\varphi^{(\lambda)}\left(u_{1}, u_{2}\right)$ is a $C^{\infty}$-function defined on a domain containing the range $\boldsymbol{u}(D)=\left\{\left(u_{1}(x), u_{2}(x)\right) ; x \in D\right\}$ and further there exists a (nonempty) domain $U \subset \Lambda=\{\lambda: \lambda \geqq 0\}$ such that if $\lambda \in U$ then all $\partial \varphi^{(\lambda)} / \partial u_{i}, \partial^{2} \varphi^{(\lambda)} / \partial u_{i} \partial u_{j}$ are bounded on $\boldsymbol{u}(D)$. In fact take linearly independent $\lambda_{1} \gg 0$ and $\lambda_{2} \gg 0$ and set for a fixed $t$

$$
u_{i}(x)=\int_{0}^{t} T_{s} f_{\lambda_{i}}(x) d s=\int_{0}^{t} e^{-\Psi_{s}\left(\lambda_{i}\right) \cdot x} d s, \quad i=1,2,
$$

then clearly $u_{i} \in D(A) \cap C^{\infty}(D)$ and by a simple calculation it is easy to see that, if $t$ is sufficiently small, the Jacobian $\partial\left(u_{1}, u_{2}\right) / \partial\left(x_{1}, x_{2}\right)$ never vanishes on some neighborhood of $D$. Hence there exists $C^{\infty}$-function $\varphi^{(\lambda)}$ such that

$$
e^{-\lambda \cdot x}=\varphi^{(\lambda)}\left(u_{1}(x), u_{2}(x)\right) .
$$

Also it is easy to see that, if $M>0$ is sufficiently large $U=\left\{\lambda=\left(\lambda_{1}, \lambda_{2}\right) ; \lambda_{i}>M\right.$, $i=1,2\}$ possesses the above property. Then by a formula on stochastic integrals (cf. [12] for diffusion processes and Kunita and Watanabe [9] for the general Hunt processes), if $\lambda \in U$,

$$
\begin{gathered}
e^{-\lambda \cdot x_{t}}-e^{-\lambda \cdot x_{0}}=\text { a martingale }+ \text { a continuous additive functional } \\
\text { of bounded variation }
\end{gathered}
$$

and hence it is clear that for each $x$ and $\lambda \in U, e^{-\psi_{t}(\lambda) \cdot x}=E_{x}\left(e^{-\lambda \cdot x_{t}}\right)$ is a function of bounded variation in $t \in[0, T]$ for every $T>0$. Thus $\psi_{t}(\lambda)$ is a function of bounded variation in $t$ and combining this with the semigroup property it is easy 
to see that $\Psi_{t}(\lambda)$ is differentiable in $t$ at $t=0$ for every $\lambda \in U_{0}$ where $U_{0}$ is a dense subset of $U$. Now the first part of Theorem 2 can be proved in exactly the same way as above and hence we have the same class of C.B.P.'s. In particular this implies that $\left\{\boldsymbol{\Psi}_{t}\right\}$ is a regular $\Psi^{2}$-semigroup.

The author wishes to thank Professor J. Lamperti for his remark.

\section{REFERENCES}

1. E. B. Dynkin, Markov processes, Springer, Berlin, 1965.

2. W. Feller, Diffusion processes in genetics, 2nd Berkeley Symposium, Univ. of Calif. Press, Berkeley, Calif., 1951, pp. 227-246.

3. G. Fichera, Sulle equazioni alle derivate parziali del secondo ordine ellittico-paraboliche, Univ. e. Politec. Torino Rend. Sem. Mat. 15 (1955-1956), 27-47.

4. R. Z. Has'minsky, Diffusion processes and elliptic differential equations degenerating at the boundary of the region, Theor. Probability Appl. 3 (1958), 430-451.

5. N. Ikeda, M. Nagasawa and S. Watanabe, Branching Markov processes, (to appear).

6. K. Ito, Lectures on stochastic processes, Tata Institute of Fundamental Research, Bombay, 1964.

7. K. Ito and H. P. McKean, Jr., Diffusion processes and their sample paths, Springer, Berlin, 1965.

8. M. Jirina, Stochastic branching processes with continuous state space, Czechoslovak Math. J. 8 (1958), 292-313.

9. H. Kunita and S. Watanabe, On square integrable martingales, Nagoya Math. J. 30 (1967), 209-245.

10. J. Lamperti, Continuous state branching processes, Bull. Amer. Math. Soc. 73 (1967), 382-386.

11. - The limit of a sequence of branching processes, $\mathrm{Z}$. Wahrscheinlichkeitstheorie und Verw. Gebiete 7 (1967), 271-288.

12. M. Motoo and S. Watanabe, On a class of additive functionals of Markov processes, J. Math. Kyoto Univ. 4 (1965), 429-469.

13. A. V. Skorokhod, Studies in the theory of random processes, (translated from Russian), Addison-Wesley, Reading, Mass., 1965.

14. H. Tanaka and M. Hasegawa, Stochastic differential equations, Seminar on Prob., Vol. 19, 1964. (Japanese)

15. A. D. Ventcel', On lateral conditions for multidimensional diffusion processes, Theor. Probability Appl. 4 (1959), 172-185.

16. S. Watanabe, On discontinuous additive functionals and Lévy measures of a Markov process, Japan J. Math. 34 (1964), 53-70.

KYOTO UNIVERSITY,

KYOTO, JAPAN

STANFORD UNIVERSITY,

Stanford, California 